\title{
EFEITOS DO TREINAMENTO RESISTIDO ASSOCIADO À OCLUSÃO VASCULAR EM LESÕES OSTEOMUSCULARES DE MEMBROS INFERIORES: REVISÃO DE LITERATURA
}

Samir Venâncio Godinho Severiano; Alan da Silva Inácio; Daniela Maria da Cruz dos Anjos; Patrícia Rabelo; Aline Ângela Silva Cruz. Revista Saúde Dinâmica, vol. 2, núm.4, 2020. Edição Especial. Faculdade Dinâmica do Vale do Piranga. 


\title{
Efeitos do treinamento resistido associado à oclusão vascular em lesões osteomusculares de membros inferiores: revisão da literatura
}

\author{
Effects of resisted training associated with vascular occlusion in osteomuscular injuries \\ of lower members: literature review
}

\author{
Samir Venâncio Godinho Severiano ${ }^{1}$, Alan da Silva Inácio ${ }^{2}$, Daniela Maria da Cruz dos Anjos ${ }^{3}$, \\ Patrícia Rabelo ${ }^{4}$, Aline Ângela Silva Cruz ${ }^{5 *}$ \\ ${ }^{1}$ Discente de Fisioterapia do Centro Universitário Estácio de Belo Horizonte, ORCID:https://orcid.org/0000-0001-8388-3272 \\ ${ }^{2}$ Discente de Fisioterapia do Centro Universitário Estácio de Belo Horizonte, ORCID: https://orcid.org/0000-0003-0282-6036 \\ ${ }^{3}$ Fisioterapeuta e Docente do Departamento de Fisioterapia do Centro Universitário Estácio de Belo Horizonte, ORCID: \\ https://orcid.org/0000-0003-4097-1787 \\ ${ }^{4}$ Docente do Departamento de Educação Física da Faculdade Pitágoras-Betim, ORCID:https://orcid.org/0000-0002-7313-6553 \\ ${ }^{5}$ Fisioterapeuta e Professora do Departamento de Fisioterapia do Centro Universitário Estácio de Belo Horizonte, ORCID: \\ https://orcid.org/0000-0002-5391-9825 \\ Autor correspondente: ninafisiobh@hotmail.com
}

\section{Resumo}

As lesões osteosmusculares de membros inferiores trazem várias limitações aos indivíduos, além de alterações que atrasam o processo de reabilitação como dor, edema, fraqueza e atrofia. Essas alterações impactam negativamente a capacidade muscular e impossibilitam que sejam aplicados programas de fortalecimento com cargas elevadas, atrasando a reabilitação. A realização do exercício resistido associado à oclusão vascular (TROV), tem por objetivo proporcionar aos indivíduos que não toleram exercícios com cargas elevadas, ganhos de força e hipertrofia muscular de forma acelerada. Verificar os efeitos do exercício resistido associado à oclusão vascular na reabilitação de lesões osteomusculares em membros inferiores. Foram selecionados artigos das bases de dados SciELO, PubMed e PEDro utilizando as palavras chaves: "vascular occlusion", "physiotherapy", "resistence exercise", "reabilitação", "oclusão vascular", "restrição do fluxo sanguíneo", "fisioterapia", "exercício resistido". Foram encontrados nove estudos. Os principais resultados observados com TR-OV foram: melhora na dor e qualidade de vida dos indivíduos avaliados, aumento na resposta hipertrófica e força. Os resultados revelaram efeitos melhores com TR-OV em comparação com a reabilitação convencional, exercícios com resistência de baixa e alta carga. Ressalta-se que o TR-OV gera resultados positivos na reabilitação em amplos aspectos como na diminuição da dor, aumento da força e massa muscular.

Palavras-chave: Fisioterapia; Oclusão Terapêutica; Reabilitação; Exercício Físico.

\begin{abstract}
Musculoskeletal injuries of the lower limbs bring several limitations to individuals, in addition to changes that delay the rehabilitation process such as pain, edema, weakness and atrophy. These changes have a negative impact on muscle capacity and make it impossible to implement strengthening programs with high loads, delaying rehabilitation. The performance of resistance exercise associated with vascular occlusion (TR-OV), aims to provide individuals who cannot tolerate exercises with high loads, strength gains and muscle hypertrophy in an accelerated way. To verify the effects of resistance exercise associated with vascular occlusion in the rehabilitation of musculoskeletal injuries in the lower limbs. Articles from the SciELO, PubMed and PEDro databases were selected using the keywords: "vascular occlusion", "physiotherapy", "resistance exercise", "rehabilitation", "vascular occlusion", "blood flow restriction”, " physiotherapy "," resistance exercise ". Nine studies were found. The main results observed with TR-OV were: improvement in pain and quality of life of the individuals evaluated, increase in the hypertrophic response and strength. The results revealed better effects with TR-OV compared to conventional rehabilitation, exercises with low and high load resistance. It is noteworthy that the TR-OV generates positive results in rehabilitation in broad aspects such as reducing pain, increasing strength and muscle mass.
\end{abstract}

Key words: Physical Therapy; Therapeutic Occlusion; Rehabilitation; Physical Exercise. 


\section{INTRODUÇÃO}

As lesões osteosmusculares de membros inferiores (MMII) trazem várias complicações para os indivíduos, dentre elas o comprometimento da função e imobilidade dos MMII. A fraqueza muscular é altamente prevalente entre a maioria das condições clínicas musculoesqueléticas no mundo todo. O efeito degenerativo do músculo atrofiado pode ser visto tanto em casos agudos como crônicos de lesões MMII, o que resulta em tratamento prolongado ou imobilização muscular, além de aumentar a incidência de fraturas e lesões ligamentares. Umas das barreiras encontradas na recuperação é a dor, fraqueza e atrofia dos músculos dos MMII, principalmente o quadríceps e isquiotibiais (VAN MELICK N et al., 2016). Este problema é comum não só apenas pela dor, mas também por causa do nível da fisiopatologia que limita movimentos funcionais e descarga de peso sobre o membro (FERRAZ et al., 2018). Dentro do processo de reabilitação, alguns indivíduos não suportam a realização de exercícios de resistência com altas cargas devido o comprometimento da lesão ocasionada, podendo em alguns casos ser até prejudicial esse esforço excessivo (KONISHI; FUKUBAYASHI, 2010).

A American College of Sports Medicine (2009) recomenda uma carga mínima de treinamento de resistência de $60 \%$ a $70 \%$ de 1 repetição máxima (1 RM) para ganhar força e $70 \%$ a $85 \%$ de 1 RM para obter hipertrofia muscular. Mas essa intensidade pode ser contraindicada para tratamentos clínicos na reabilitação de lesões de MMII (THOMAS et al., 2013). Na maioria dos casos, os indivíduos que não conseguem realizar esse treinamento são os que mais necessitam de ganhos de massa muscular e força, devido a fraqueza muscular e atrofia causada pela fisiopatologia, bem como precisam obter aumento da sua capacidade muscular para melhora do quadro clínico. Por outro lado, uma ferramenta de trabalho para os fisioterapeutas é o programa de reabilitação com resistência de baixa carga, mas a menos que seja usado até a fadiga muscular, que é pouco suportada por esses indivíduos, será improdutivo em provocar aumentos na hipertrofia imediata (PATTERSON et al., 2017).

Alguns estudos têm achados de permanência de fraqueza do quadríceps por muitos meses ou até anos após a reconstrução do ligamento cruzado anterior (LCA), o que prejudica o retorno ao fisiológico quando comparado ao lado contralateral (PALMIERI-SMITH; THOMAS; WOJTY, 2008). Isso acontece mesmo com intervenção precoce de fisioterapia, com medidas 
preventivas, estimulação com eletroterapia, fortalecimento em cadeia cinética aberta (CCA) e cadeia cinética fechada (CCF) e sustentação do próprio peso (INGERSOLL et al., 2008).

O treinamento de oclusão vascular, mais conhecido como KAATSU, tem por objetivo proporcionar aos indivíduos que não toleram exercícios de altas cargas de 1 RM, hipertrofia muscular de forma acelerada. Com a oclusão vascular ocorre uma hipóxia temporária no músculo, além da produção de metabólitos como lactato e íons H+. Essas alterações fisiológicas geram uma sinalização para o sistema nervoso central, e como consequência, na recuperação ocorre maior liberação de hormônios anabólicos. Além disso, o reduzido aporte sanguíneo decorrente da oclusão desencadeia uma fadiga precoce das fibras tipo I, o que ocasiona um maior recrutamento de fibras de contração rápida (tipo II) que possuem maior resposta hipertrófica (LOENNEKE et al., 2009).

O procedimento implica na aplicação de uma braçadeira no estilo torniquete na região proximal do membro imediatamente antes do exercício (NICHOLAS N. DEPHILLIPO et al. 2018). Na sequência, são realizados exercícios resistidos com cargas leves ou moderadas produzindo reações metabólicas na musculatura e promovendo ganhos comparados a exercícios de intensidades de cargas mais altas, como 80\% RM (NICHOLAS N. DEPHILLIPO et.al. 2018). Na literatura existem estudos do método de treinamento com oclusão vascular, alegando que o torque e a hipertrofia muscular podem melhorar com exercício de baixa resistência se associados a restrição de fluxo sanguíneo local (SATO et al. 2015). No entanto, ainda são necessárias mais evidências científicas para identificar quais seriam os benefícios do método na reabilitação realizada pela fisioterapia. $\mathrm{O}$ intuito desse estudo é identificar os ganhos relatados na literatura e o mecanismo que ocorre durante os exercícios com restrição do fluxo sanguíneo. A partir dos resultados apresentados, almeja-se contribuir para a compreensão do método. Assim, o objetivo do presente estudo foi verificar, através de uma revisão de literatura, os efeitos do exercício resistido associado com a oclusão vascular na reabilitação de pacientes com lesões osteomusculares em MMII.

\section{MATERIAIS E MÉTODOS}

Esta pesquisa foi realizada através de uma busca na literatura nas bases de dados eletrônica: SciELO, PubMed e PEDro. As palavras chaves foram: "vascular occlusion", 
"physiotherapy", "resistence exercise", "rehabilitatin" "reabilitação", "oclusão vascular", "restrição do fluxo sanguíneo", "fisioterapia" e "exercício resistido".

A pesquisa foi limitada as línguas portuguesa e inglesa, com estudos realizados com humanos adultos sedentários de 18 a 60 anos. Os critérios de inclusão foram: ambos os sexos, acima de 18 anos, com uso de oclusão vascular durante o exercício resistido para reabilitação de lesões osteomusculares em MMII, pós-operatório de lesões osteomusculares. Nos critérios de exclusão foram: artigos de revisão, pacientes portadores de distúrbios neurológicos, artigos que estão associados com outras técnicas para hipertrofia e ganho de força.

A seleção foi realizada com intervalo de busca dos artigos do ano de 2000 até setembro de 2019, a triagem dos artigos foi da seguinte forma: primeiramente, foi realizado a leitura do título e do resumo, ao final, foi realizado a leitura do artigo na integra.

\section{RESULTADOS}

O processo de busca inicial obteve 137 estudos. Depois disso, títulos foram lidos e foram excluídos 68 estudos, então foram lidos os resumos dos demais estudos, dos quais 14 revisões foram excluídas. Posteriormente, os artigos foram separados e, após a realização da leitura completa, 46 foram excluídos por apresentarem uma metodologia não condizente com os critérios de inclusão. Os 9 artigos restantes foram considerados elegíveis de acordo com os critérios previamente definidos, sendo lesões de MMII, idade e em humanos sedentários. O processo da pesquisa está representado na figura 1. 
Figura 1 Fluxograma do processo de seleção do estudo.

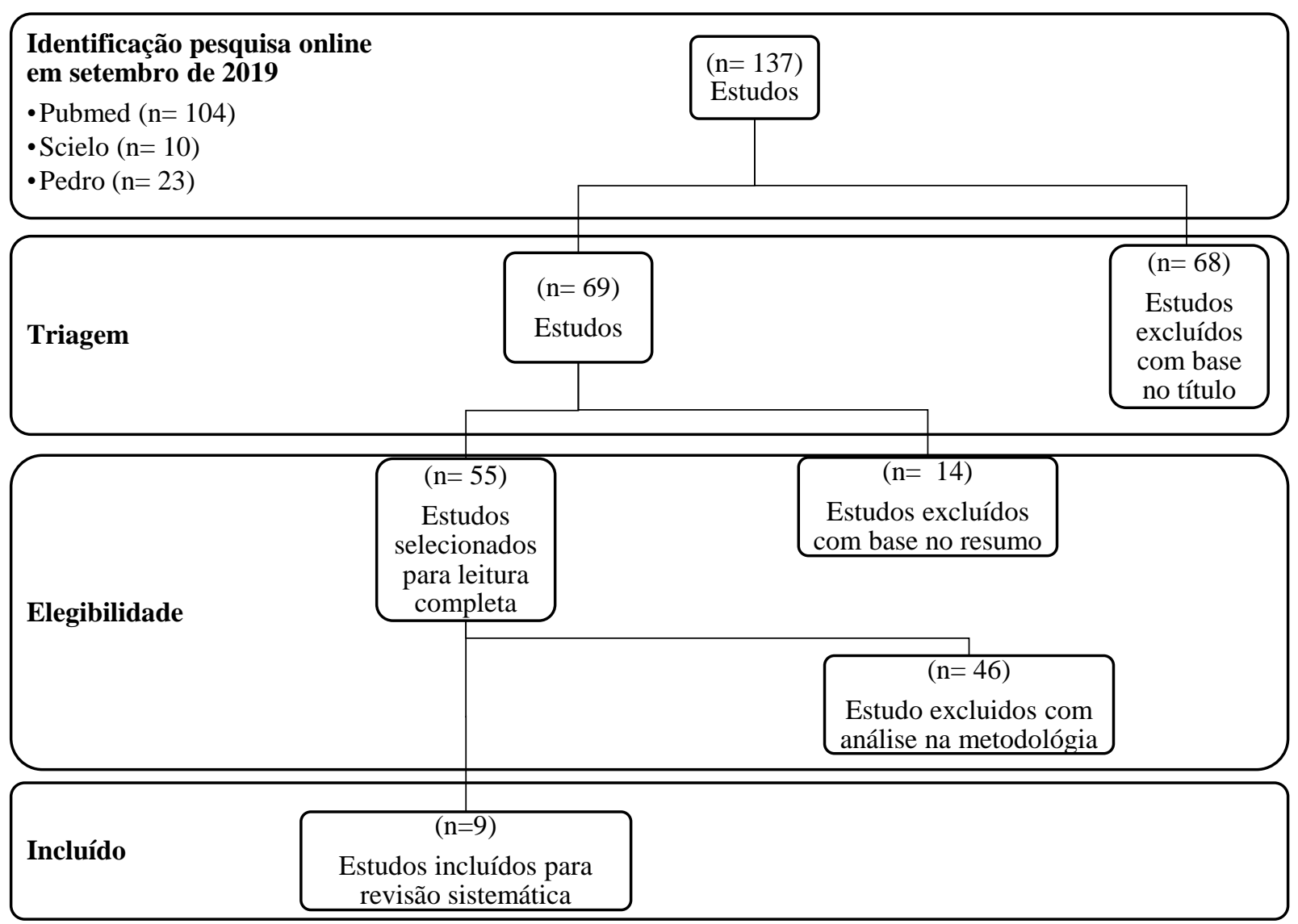

Dois autores separadamente avaliaram todos os artigos e extraíram os seguintes dados: amostra, desenho do estudo (instrumento de medida, desfecho avaliado, intervenção) e resultados. A avaliação dos resultados da resposta hipertrófica, força, dor e função física foram $100 \%$ extraídos. Posteriormente, todos os estudos foram cruzados para confirmar a precisão. Os dados extraídos estão disponíveis na Tabela 1.

Foram encontrados nove estudos $(100 \%)$ que se enquadraram nos critérios estabelecidos. Os estudos que avaliaram o efeito do treinamento resistido realizado com oclusão vascular reportaram as seguintes condições de saúde: osteoartrite $(\mathrm{OA})$ de joelho $(33,33 \%)$, dor anterior no joelho ou dor patelofemoral (22,22\%), pós-operatório de reconstrução de LCA $(22,22 \%)$, pós artroscopia de joelho $(11,11 \%)$, e pós-operatório de ruptura de tendão de Aquiles $(11,11 \%)$. 
Treinamento resistido associado à oclusão vascular

Tabela 1 Protocolos utilizados nos estudos selecionados.

\begin{tabular}{|c|c|c|c|c|c|c|c|c|}
\hline $\begin{array}{l}\text { Autor } \\
\text { (Data) }\end{array}$ & $\begin{array}{c}\text { Desenho } \\
\text { do Estudo }\end{array}$ & Amostra & $\begin{array}{l}\text { Instrumento } \\
\text { de medida }\end{array}$ & $\begin{array}{c}\text { Pressão de } \\
\text { oclusão vascular }\end{array}$ & Desfecho & Protocolo & $\begin{array}{l}\text { Duração e } \\
\text { frequência }\end{array}$ & Resultado \\
\hline $\begin{array}{l}\text { Bryk et } \\
\text { al., } 2016\end{array}$ & ECR & $\begin{array}{c}\text { G1: } \mathrm{n}=17 \\
\text { (convenciona } \\
\text { 1) } \\
\text { G2: } \mathrm{n}=17 \\
(\mathrm{TR}-\mathrm{OV}) \\
\text { Sexo: } \\
\text { Feminino } \\
\text { Idade: Média } \\
60 \text { anos }\end{array}$ & $\begin{array}{c}\text { NPRS: } \\
\text { Avaliação da } \\
\text { dor } \\
\text { Lequesne: } \\
\text { Avaliação da } \\
\text { dor, } \\
\text { desconforto e } \\
\text { função. } \\
\text { TUG: Avalia } \\
\text { mobilidade } \\
\text { física e } \\
\text { equilibro } \\
\text { Força } \\
\text { muscular: } \\
\text { Dinamômetro } \\
\text { portátil }\end{array}$ & $200 \mathrm{mmHg}$. & $\begin{array}{c}\text { Força de } \\
\text { quadríceps, } \\
\text { alívio da dor e } \\
\text { melhora } \\
\text { funcional. }\end{array}$ & $\begin{array}{l}\text { Protocolo=G1: } \\
\text { alongamento e } \\
\text { fortalecimento de } \\
\text { MMII, exercício } \\
\text { de quadríceps } \\
\text { combinado com } \\
\text { alta carga de } 1 \\
\text { RM (70\%). } \\
\text { G2: alongamento } \\
\text { e fortalecimento } \\
\text { de MMII, } \\
\text { exercício de } \\
\text { quadríceps } \\
\text { combinado com } \\
\text { PVO com baixa } \\
\text { caga de } 1 \text { RM } \\
\text { (30\%). }\end{array}$ & $\begin{array}{c}18 \text { sessões, } 3 x \\
\text { por semana, } \\
\text { total de } 6 \\
\text { semanas. }\end{array}$ & $\begin{array}{c}\text { Força muscular: Não } \\
\text { houve diferença } \\
\text { significativa em G1 } \\
\text { e G2. } \\
\text { Dor e função: Não } \\
\text { houve diferença } \\
\text { significativa em G1 } \\
\text { e G2 } \\
\text { Equilíbrio: Não } \\
\text { houve diferença } \\
\text { significativa em G1 } \\
\text { e G2 } \\
\text { Dor: Melhora do } \\
\text { desconforto durante } \\
\text { o exercício de } \\
\text { quadríceps em G2 }\end{array}$ \\
\hline $\begin{array}{l}\text { Ferraz et } \\
\text { al., } 2018\end{array}$ & ECR & $\begin{array}{l}\text { HI-RT: } n=16 \\
\text { LI-RT: n= } 16\end{array}$ & $\begin{array}{c}\text { Oclusão } \\
\text { vascular: } \\
\text { manguito de ar } \\
\text { proximal a } \\
\text { coxa } \\
1 \text { RM: Força } \\
\text { muscular }\end{array}$ & $\begin{array}{c}70 \% \text { da pressão } \\
\text { de oclusão total } \\
\text { de cada }\end{array}$ & $\begin{array}{c}\text { Força } \\
\text { muscular e } \\
\text { hipertrofia de }\end{array}$ & $\begin{array}{c}\text { Protocolo= HI- } \\
\text { RT 4x 10rep.a } \\
50 \% \text { de } 1 \text { RM } \\
\text { LI-RT 4x e 15rep. } \\
\text { A } 20 \% .\end{array}$ & 12 semanas, $2 \mathrm{x}$. & $\begin{array}{c}\text { Força muscular: } \\
\text { Aumento em } \\
\text { extensão de joelho } \\
\text { em HI-RT e BFRT. } \\
\text { Aumento da área de } \\
\text { secção transversa }\end{array}$ \\
\hline
\end{tabular}

SAÚDE DINÂMICA - Revista Científica Eletrônica

6a Edição 2020 | Ano II, no 4 | ISSN-2675-133X 
Treinamento resistido associado à oclusão vascular

\begin{tabular}{|c|c|c|c|c|c|c|c|c|}
\hline & & $\begin{array}{c}\text { BFRT:n=16 } \\
\text { (TR-OV) } \\
\text { Sexo: } \\
\text { Feminino } \\
\text { Idade: Média } \\
60 \text { anos }\end{array}$ & $\begin{array}{c}\text { Função física: } \\
\text { Testes TST e } \\
\text { TUG. } \\
\text { Área de secção } \\
\text { transversa do } \\
\text { quadríceps: } \\
\text { tomografia } \\
\text { computadoriza } \\
\text { da } \\
\text { Dor e } \\
\text { qualidade de } \\
\text { vida: } \\
\text { WOMAC }\end{array}$ & $\begin{array}{c}\text { indivíduo (média } \\
\text { de } 160 \text { a } 200 \\
\mathrm{mmHg} \text {. }\end{array}$ & $\begin{array}{l}\text { membros } \\
\text { inferiores. }\end{array}$ & $\begin{array}{c}\text { Após a segunda } \\
\text { semana, HI-RT } \\
\text { evoluiu para } 80 \% \\
\text { e LI-RT evoluiu } \\
\text { para 30\% }\end{array}$ & & $\begin{array}{c}\text { nos grupos HI-RT e } \\
\text { BFRT. } \\
\text { Dor e qualidade de } \\
\text { vida: Diminuição do } \\
\text { score nos grupos LI- } \\
\text { RT e BFRT. } \\
\text { Função física } \\
\text { (TUG): Não houve } \\
\text { diferença } \\
\text { significativa nos } \\
\text { grupos HI-RI, BFRT } \\
\text { e LI-RT. } \\
\text { Função física (TST): } \\
\text { Melhora em HI-RI e } \\
\text { BFRT. }\end{array}$ \\
\hline $\begin{array}{l}\text { Giles et } \\
\text { al., } 2017\end{array}$ & ECA & $\begin{array}{l}\text { Standard: } \mathrm{n}= \\
\qquad 34 \\
\text { BFR: } \mathrm{n}=35 \\
\text { (TR-OV) } \\
\text { Sexo: } \\
\text { Masculino e } \\
\text { Feminino } \\
\text { Idade: } 27\end{array}$ & $\begin{array}{c}\text { Dinamômetro: } \\
\text { Força } \\
\text { muscular do } \\
\text { quadríceps } \\
\text { Ultrassom: } \\
\text { Espessura do } \\
\text { músculo } \\
\text { quadríceps } \\
\text { Oclusão } \\
\text { vascular: } \\
\text { manguito } \\
\text { proximal a } \\
\text { coxa }\end{array}$ & $\begin{array}{c}60 \% \text { da pressão } \\
\text { de oclusão total } \\
\text { de cada } \\
\text { indivíduo. }\end{array}$ & $\begin{array}{c}\text { Força e } \\
\text { hipertrofia de } \\
\text { quadríceps. }\end{array}$ & $\begin{array}{l}\text { Ambos os grupos: } \\
5 \text { min de bicicleta } \\
\text { de exercício de } \\
\text { intensidade leve. } \\
\text { Standard: } \\
\text { extensor de joelho } \\
\text { com carga } \\
\text { máxima } 3 x \text { 7-10 } \\
\text { repetições } \\
\text { máximas. } \\
\text { BFR: } 30 \% \text { de } 1 \\
\text { RM, 3x, } 15 \text { rep. }\end{array}$ & $\begin{array}{c}3 \text { vezes por } \\
\text { semana, } 6 \text { meses } \\
\text { de tratamento. }\end{array}$ & $\begin{array}{l}\text { Melhor diminuição } \\
\text { da dor em } 8 \text { semanas } \\
\text { no grupo BFR. } \\
\text { Aumento da } \\
\text { espessura do } \\
\text { quadríceps no grupo } \\
\text { normalizado em } 8 \\
\text { semanas. } \\
\text { Melhora força de } \\
\text { quadríceps em BFR } \\
\text { e Standard. }\end{array}$ \\
\hline
\end{tabular}


Treinamento resistido associado à oclusão vascular

\begin{tabular}{|c|c|c|c|c|c|c|c|c|}
\hline $\begin{array}{c}\text { Inversen } \\
\text { et al., } \\
2014\end{array}$ & ECR & $\begin{array}{c}\text { G1 (TR-OV) } \\
\text { n=12 } \\
\text { G2 (controle) } \\
\text { n= } 12 \\
\text { Sexo: } \\
\text { Feminino e } \\
\text { Masculino } \\
\text { Idade: Média } \\
27.35\end{array}$ & $\begin{array}{c}\text { Oclusão } \\
\text { vascular: } \\
\text { manguito } \\
\text { portátil } \\
\text { Pressão } \\
\text { 180mmHg } \\
\text { Software } \\
\text { incluído no } \\
\text { sectra PACS: } \\
\text { Área de secção } \\
\text { transvers0a }\end{array}$ & $\begin{array}{c}130 \text { a } 180 \\
\text { mmHg. }\end{array}$ & $\begin{array}{c}\text { Menor } \\
\text { redução na } \\
\text { área de secção } \\
\text { transversa do } \\
\text { quadríceps. }\end{array}$ & $\begin{array}{l}\text { G1: isometria de } \\
\text { quadríceps em } \\
\text { extensão de } \\
\text { joelho com } \\
\text { oclusão vascular } \\
\text { 10x } 5 \text { minutos. } \\
\text { G2: isometria de } \\
\text { quadríceps em } \\
\text { extensão de } \\
\text { joelho 20x } 5 \\
\text { minutos }\end{array}$ & $\begin{array}{c}18 \text { dias, } 2 \mathrm{x} \text { por } \\
\text { dia. }\end{array}$ & $\begin{array}{c}\text { Área de secção } \\
\text { transversa: } \\
\text { Diminuição do } \\
\text { quadríceps em G1 e } \\
\text { G2 } \\
\text { Não houve diferença } \\
\text { estatística entre } \\
\text { homens e mulheres }\end{array}$ \\
\hline $\begin{array}{c}\text { Korakaki } \\
\text { s et al., } \\
2018 .\end{array}$ & ECRP & $\begin{array}{l}\text { LLRT-BFR } \\
(\mathrm{n}=20) \\
(\mathrm{TR}-\mathrm{OV}) \\
\text { LLRT } \\
(\mathrm{n}=20) \\
\text { Média de } \\
\text { 29,7 anos, } \\
\text { entre } 18 \text { e } 65 \\
\text { anos, do sexo } \\
\text { masculino. }\end{array}$ & $\begin{array}{c}\text { NRS: } \\
\text { Avaliação da } \\
\text { dor } \\
\text { SLS e SDT: } \\
\text { Teste } \\
\text { funcional } \\
\text { Oclusão } \\
\text { vascular: } \\
\text { Manguito de } \\
\text { BFR (TR-OV) }\end{array}$ & $\begin{array}{c}80 \% \text { da pressão } \\
\text { de oclusão total } \\
\text { de cada } \\
\text { indivíduo. }\end{array}$ & $\begin{array}{c}\text { Redução da } \\
\text { dor. }\end{array}$ & $\begin{array}{l}\text { LLRT-BFR: } \\
\text { extensão de } \\
\text { joelho em CCA: } \\
\text { 3x 15rep } \\
\text { LLRT-BFR: } \\
\text { extensão de } \\
\text { joelho em CCA } \\
\text { com utilização do } \\
\text { manguito: } 3 x \\
\text { 15rep } \\
\text { Ambos os grupos: } \\
\text { treinamento de } \\
\text { resistência em } \\
\text { MMII, exercícios }\end{array}$ & $3 x$ 15rep & $\begin{array}{l}\text { Dor: Diminuição } \\
\text { imediata e pós } \\
\text { fisioterapia em } \\
\text { LLRT-BFR nos } \\
\text { SLSS, SLSD e SDT } \\
\text { Não houve diferença } \\
\text { significativa em } \\
\text { LLRT }\end{array}$ \\
\hline
\end{tabular}


Treinamento resistido associado à oclusão vascular

\begin{tabular}{|c|c|c|c|c|c|c|c|c|}
\hline & & & & & & $\begin{array}{l}\text { do core e } \\
\text { equilíbrio. }\end{array}$ & & \\
\hline \multirow[t]{2}{*}{$\begin{array}{l}\text { Ohta et } \\
\text { al., } 2003\end{array}$} & PCR & $\begin{array}{c}\text { GN: } \mathrm{n}=22 \\
\text { (convenciona } \\
1) \\
\text { GR: } \mathrm{n}=22 \\
(\mathrm{TR}-\mathrm{OV}) \\
\text { Média de } 29 \\
\text { anos, entre } 18 \\
\text { e } 52 \text { anos, do } \\
\text { sexo } \\
\text { feminino e } \\
\text { masculino. }\end{array}$ & $\begin{array}{c}\text { Oclusão } \\
\text { vascular: } \\
\text { Torniquete } \\
\text { Miodinômetro } \\
\text { isocinetico: } \\
\text { Força } \\
\text { muscular de } \\
\text { extensores e } \\
\text { flexores de } \\
\text { joelho } \\
\text { Ressonância } \\
\text { magnética: } \\
\text { Área de secção } \\
\text { transversa } \\
\text { Diâmetro de } \\
\text { fibra muscular: } \\
\text { Coleta dos } \\
\text { músculos } \\
\text { vastos laterais } \\
\text { durante a } \\
\text { cirurgia. }\end{array}$ & $180 \mathrm{mmHg}$. & $\begin{array}{l}\text { Força e } \\
\text { hipertrofia } \\
\text { muscular. }\end{array}$ & $\begin{array}{l}\text { 1 semana } \\
\text { GN/GR: abdução } \\
\text { e flexão de } \\
\text { quadril 5s, } 20 \text { rep. } \\
\text { Adução de } \\
\text { quadril e } \\
\text { quadríceps 5s, } 20 \\
\text { rep. } \\
\text { 6rep. } \\
\text { Step-up 20rep. } \\
\text { Tubo elástico } \\
\text { joelho } 45^{\circ} \text { a } 100^{\circ} \\
\text { graus 20rep. } \\
\text { 2 semanas: GR: } \\
\text { inclusão da } \\
\text { oclusão vascular. }\end{array}$ & $\begin{array}{c}16 \text { semanas após } \\
\text { a reconstrução } \\
\text { do LCA }\end{array}$ & $\begin{array}{c}\text { Força muscular: } \\
\text { Aumento nos } \\
\text { músculos flexores e } \\
\text { extensores de joelho } \\
\text { em GN e GR. } \\
\text { Área de secção } \\
\text { transversa: Aumento } \\
\text { do extensor de } \\
\text { joelho em GR. } \\
\text { Diâmetro de fibra } \\
\text { muscular: Não houve } \\
\text { diferença } \\
\text { significativa para } \\
\text { GN e GR. }\end{array}$ \\
\hline & & $\begin{array}{c}\text { G: PBFR }(n= \\
\text { 19) TR-OV }\end{array}$ & $\begin{array}{c}\text { Kaatsu Master } \\
\text { BFR: Proximal } \\
\text { a coxa }\end{array}$ & & & $\begin{array}{l}\text { Os dois grupos } \\
\text { realizaram } \\
\text { contração } \\
\text { isotônica bilateral }\end{array}$ & & \\
\hline
\end{tabular}


Treinamento resistido associado à oclusão vascular

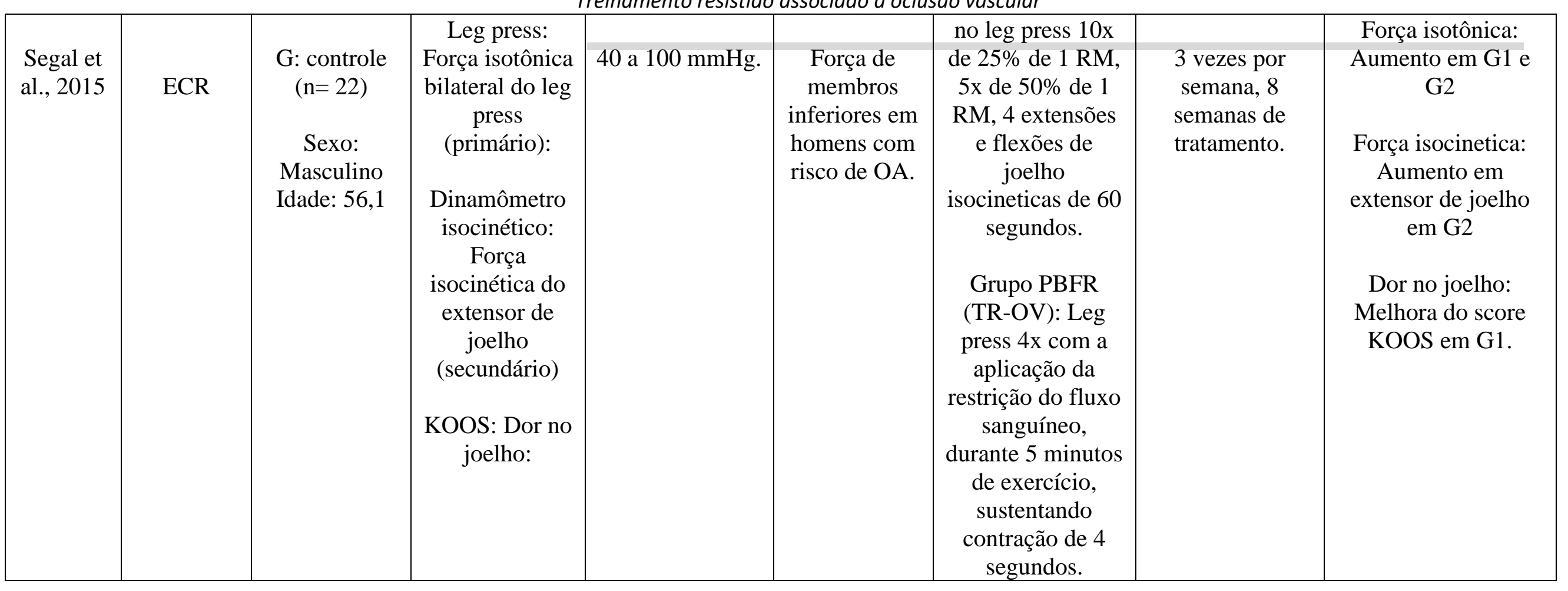


Treinamento resistido associado à oclusão vascular

\begin{tabular}{|c|c|c|c|c|c|c|c|c|}
\hline $\begin{array}{l}\text { Tennent } \\
\text { et al., } \\
2017\end{array}$ & ECR & $\begin{array}{l}\text { G1 }(\mathrm{TR}-\mathrm{OV}) \\
\mathrm{n}=11 \\
\text { randomizado } \\
\mathrm{G} 2 \\
\text { (controle) } \\
\mathrm{n}=13 \\
\text { randomizado } \\
\text { Sexo: } \\
\text { Masculino e } \\
\text { feminino } \\
\text { Idade: média } \\
37\end{array}$ & $\begin{array}{c}\text { Torniquete } \\
\text { portátil: } \\
\text { oclusão } \\
\text { vascular } \\
\text { (proximal a } \\
\text { coxa) } \\
\text { SSWV, STS5, } \\
\text { FSST, TSA } \\
\text { :Desempenho } \\
\text { físico } \\
\text { KOOS: Dor e } \\
\text { função física } \\
\text { Dinamômetro: } \\
\text { Teste de força } \\
\text { Ultra- } \\
\text { sonografia } \\
\text { Duplex: } \\
\text { Circunferência } \\
\text { da coxa }\end{array}$ & $\begin{array}{c}80 \% \text { da pressão } \\
\text { de oclusão total } \\
\text { de cada } \\
\text { indivíduo. }\end{array}$ & $\begin{array}{c}\text { Força } \\
\text { muscular e } \\
\text { hipertrofia de } \\
\text { quadríceps }\end{array}$ & $\begin{array}{l}\text { Ambos os grupos } \\
\text { foram submetidos } \\
\text { a fisioterapia com } \\
\text { protocolo de } \\
\text { artroscopia não } \\
\text { reconstrutiva de } \\
\text { joelho. } \\
\text { G2: leg press, } \\
\text { extensão saboreia } \\
\text { e prensa de pernas } \\
\text { reversa em pé. } \\
1 \times 30 s, 3 x 15 s\end{array}$ & $\begin{array}{c}12 \text { sessões, } 6 \\
\text { semanas. }\end{array}$ & $\begin{array}{l}\text { Circunferência da } \\
\text { coxa: Melhora } \\
\text { significativa em G1 } \\
\text { Dor e função física: } \\
\text { Melhora no score da } \\
\text { subescalas em G2 } \\
\text { Desempenho físico } \\
\text { : Melhora nas } \\
\text { escalas de } \\
\text { desempenho físico } \\
\text { em G1 } \\
\text { Força muscular: } \\
\text { Aumento } \\
\text { significativo em G1 }\end{array}$ \\
\hline & & 2 indivíduos & $\begin{array}{c}\text { Paciente 1: } \\
\text { força de } \\
\text { tornozelo: } \\
\text { isocinético } \\
\text { biodex system }\end{array}$ & $\begin{array}{l}80 \% \text { da pressão } \\
\text { de oclusão total }\end{array}$ & & $\begin{array}{l}\text { Paciente 1: Leg } \\
\text { press } 90 \text { graus de } \\
\text { flexão: 1x, 30rep, } \\
\text { 3x, 15rep. } \\
\text { Panturrilha: 1x, } \\
\text { 30rep, 3x, 15rep. }\end{array}$ & $\begin{array}{c}\text { Paciente 1: } 5 \\
\text { semanas de } \\
\text { tratamento. }\end{array}$ & $\begin{array}{c}\text { Paciente 1: Aumento } \\
\text { da força de flexão } \\
\text { plantar. }\end{array}$ \\
\hline
\end{tabular}

SAÚDE DINÂMICA - Revista Científica Eletrônica

6a Edição 2020 | Ano II, no 4 | ISSN-2675-133X 
Treinamento resistido associado à oclusão vascular

\begin{tabular}{|c|c|c|c|c|c|c|c|c|}
\hline $\begin{array}{l}\text { Yow et } \\
\text { al., } 2017\end{array}$ & RL & $\begin{array}{c}\text { Sexo: } \\
\text { Masculino } \\
\text { Idade: } 33,5 .\end{array}$ & $\begin{array}{c}\text { Oclusão } \\
\text { vascular: } \\
\text { torniquete } \\
\text { Paciente 2: } \\
\text { força } \\
\text { muscular: } \\
\text { isocinetico } \\
\text { basal } \\
\text { Oclusão } \\
\text { vascular: } \\
\text { torniquete }\end{array}$ & $\begin{array}{c}\text { de cada } \\
\text { indivíduo. (180 } \\
\text { mmHg.) }\end{array}$ & $\begin{array}{c}\text { Força, } \\
\text { potência e } \\
\text { função do } \\
\text { tríceps sural. }\end{array}$ & $\begin{array}{l}\text { Ambos com } 30 \% \\
\text { de 1RM. } \\
\text { Paciente 2: Leg } \\
\text { press 90• graus de } \\
\text { flexão: 1x, 30rep, } \\
\text { 3x, 15rep. } \\
\text { Panturrilha: 1x, } \\
\text { 30rep, 3x, 15rep. } \\
\text { Ambos com } \\
\text { 30\% de 1RM. }\end{array}$ & $\begin{array}{c}\text { Paciente 2: } 6 \\
\text { semanas de } \\
\text { tratamento. }\end{array}$ & $\begin{array}{c}\text { Aumento da potência } \\
\text { muscular. } \\
\text { Deambulação sem } \\
\text { dispositivo de } \\
\text { auxílio. } \\
\text { Paciente } 2 \text { : Aumento } \\
\text { da força de flexão } \\
\text { plantar. } \\
\text { Aumento da potência } \\
\text { muscular. } \\
\text { Retorno a prática } \\
\text { esportiva. }\end{array}$ \\
\hline
\end{tabular}

Legenda: 1 RM: Uma repetição máxima; ACSA: Anatomical cross sectional área; BFRT: Blood flow restriction; TR-OV: Exercício resistido associado a oclusão vascular; CCA: Cadeia cinética aberta; ECR: Ensaio clínico randomizado/Estudo de caso randomizado; ECRP: Estudo controlado randomizado piloto; FSST: Teste de 4 passos quadrados; G: Grupo. GN: Grupo convencional; GR: Grupo oclusão vascular; HI-RT: High intensity resistance training; KNOO: Knee injury and Osteoarthritis Outcome Score; LCA: Ligamento cruzado anterior; LI-RT: Low high intensity resistance training; LLRT: Low load resistance training; MMII: Membros inferiores; NRS: Numeric rating scale; OA: Osteoartrite; PBFR: Partial Blood flow restriction; PCR: Prospectivo controle randomizado; PVO: Partial vascular occlusion; RM: Repetições máximas; Rep: Repetições; RL: Relato de caso; SDT: Teste de redução SLSs: Agachamento raso; SLSd: Agachamento profundo; SSWV: Velocidade de caminhada auto-selecionada; STS5: Sit to stand; TSA: Subida temporizada da escada; TST: Timed Stair Test; TUG- Time Up and Go; WOMAC: Westerm Ontario and McMaster Universities X: vezes. 
Bryk et al., (2016), Ferraz et al., (2018), Segal et al., (2015), Giles et al., (2017), e Korakakis et al., (2018) investigaram exercícios com oclusão vascular parcial em pacientes com OA do joelho e dor anterior do joelho (dor patelofemoral), comparando os grupos, fisioterapia convencional altas cargas $70 \% \mathrm{RM}$, baixas cargas $30 \%$ a $50 \% \mathrm{RM}$, e exercício resistido combinado com oclusão vascular com baixa carga (TR-OV) $30 \%$ a 50\% de 1 RM. Ao usar baixas cargas, o grupo TR-OV induziu menos estresse articular resultando em diminuição do desconforto e da dor na região anterior do joelho nos cinco estudos acima, e tanto os grupos controle (alta carga) quanto os grupos TR-OV obtiveram melhoras na força e resposta hipertrófica.

Inversen et al. (2014) compararam o efeito de exercícios isométricos de baixas cargas com e sem oclusão vascular no tratamento pré e pós-operatório de reconstrução de LCA. Importante ressaltar que o grupo experimental realizou os exercícios nos intervalos da oclusão vascular. Os autores observaram que não ocorreu melhora significativa em nenhum dos grupos. Os demais estudos que utilizaram exercício isotônicos com oclusão vascular, relataram hipertrofia, aumento da força e melhora da dor como observado no estudo Ohta et al., (2003) que investigaram os efeitos do exercício isotônico com e sem oclusão vascular no pósoperatório de reconstrução de LCA e identificou aumento do diâmetro das fibras tipo 1 e tipo 2 no grupo com oclusão vascular.

Tennent et al. (2017) investigaram os efeitos do treinamento resistido com oclusão vascular no pós artroscopia de joelho comparado com exercício convencional. Foi identificado melhora da dor e força muscular nos dois grupos, mas o grupo TR-OV obteve uma melhora maior nos testes funcionais e de força quando comparado ao grupo controle. Além disso, apenas o grupo TR-OV obteve aumento significativo na circunferência da coxa.

Yow et al., (2017), pesquisaram os efeitos da oclusão vascular com exercícios resistidos no pós operatório de ruptura de tendão de Aquiles em dois pacientes. Os autores identificaram melhora da força e potência muscular dos flexores plantar de forma acelerada.

Indivíduos com lesão ligamentares, osteoarticulares e cirurgias apresentam redução do movimento, redução da massa muscular e força, o que prejudica o seu desempenho funcional (DE JONG et al., 2007). No entanto, na reabilitação nem sempre é possível a aplicação de exercício com alta carga nas fases iniciais quando a capacidade funcional do indivíduo está reduzida. Intervenções com o objetivo de promover o aumento da força e massa muscular 
(hipertrofia) nos momentos iniciais da reabilitação são importantes para acelerar o processo de recuperação nesse sentido. Os resultados dos estudos sugerem que o treinamento resistido associado com a oclusão vascular pode ser uma alternativa eficaz no tratamento precoce dos pacientes com lesões de MMII.

\section{DISCUSSÃO}

Este estudo teve o objetivo de analisar os efeitos da oclusão vascular associado com exercício resistido de baixa carga como reabilitação de MMII. As recentes pesquisas sugerem que o TR-OV com baixa carga produz aumento da força e hipertrofia muscular (HEITKAMP HC 2015). O treinamento TR-OV foi eficaz e verdadeiro tanto quanto os efeitos de treinamento resistido de carga máxima, embora o grau de aumento hipertrófico e força possam apresentar uma individualidade biológica (LIXANDRÃO et al., 2018). Portanto, podemos utilizar essa técnica em pacientes com lesões osteomusculares em MMII em reabilitação clínica, já que os mesmos pacientes são contraindicados ao treino com altas cargas devido à pressão e sobrecarga gerada em cima das articulações e músculos.

Para utilizar técnicas de reabilitação devemos entender o processo que ocorre nas lesões osteomusculares, e quais os fatores intrínsecos e extrínsecos podem influenciar na recuperação. Nos fatores intrínsecos estão aqueles relacionados a fatores individuais, como capacidade muscular de força e flexibilidade, anatomia e biomecânica, fatores nutricionais e emocionais. Já os fatores extrínsecos estão relacionados ao ambiente: modalidade ou atividade diária, duração da atividade, utilização de materiais de proteção durante atividades e traumas (FERNANDES et al., 2011).

Quando a demanda está acima da capacidade do tecido muscular, pode ocasionar um desgaste tecidual, já que o mesmo não está capacitado para suportar tal demanda (GUILHERME F. REIS et al., 2015). Como consequência, nota-se um agravamento ou surgimento de uma lesão no tecido ou nas estruturas afetadas. A fraqueza muscular é altamente prevalente nas condições clínicas de lesões musculoesqueléticas. Os efeitos degenerativos da atrofia muscular podem ser vistos com lesões agudas e crônicas nas lesões musculoesqueléticas, o que resulta em um tratamento prolongado ou imobilização muscular (THOMAS et al., 2016). E a perda de força é um importante fator de risco para OA (PETTERSON et al., 2010). 
Entretanto, a partir da compreensão dos efeitos de uma lesão osteomuscular e da técnica de oclusão vascular pode-se analisar o uso dessa técnica nas lesões em MMII.

No entanto, há uma falta de consistência e padronização em relação aos protocolos TROV para reabilitação, portanto, programas definitivos não podem ser determinados. Porém o treinamento com TR-OV pode ser benéfico se incorporado aos planos de reabilitação. Os artigos abordados nesta revisão mostram que a oclusão vascular pode ser associada tanto com exercícios em CCA quanto em CCF para obter resultados na reabilitação, mas não está definido o protocolo de volume e frequência.

Um achado importante foi que $100 \%$ dos estudos que avaliaram a dor identificaram resultados positivos. Segundo Ferraz et al. (2018) esse resultado acontece devido a diminuição de estresse articular durante o exercício com TR-OV e adaptação da musculatura envolvida, assim gerando menos sobrecarga no tecido lesionado. No estudo de Bryk et al. (2016), os autores relataram como principal benefício do exercício com TR-OV a diminuição do desconforto em pacientes com OA de joelho, e apresentou melhora na dor e sobre a resposta hipertrófica e força obteve os mesmos resultados em termos de adaptações musculares funcionais quando comparado a exercícios de altas cargas, foi utilizado exercícios de CCA em ambos os grupos e carga no grupo TR-OV de 30\% de 1RM. Já no estudo de Ferraz et al., (2018), e Segal et al., (2015) avaliaram também pacientes com OA de joelho e utilizaram exercícios em CCA e CCF em ambos os grupos. Os dois estudos com carga no grupo TR-OV de 30\% de 1RM, e em concordância nos estudos Bryk et al., (2016), Ferraz et al., (2018), e Segal et al., (2015) com pacientes com OA de joelho, houve melhora da dor durante a funcionalidade dos pacientes. O estudo de Giles et al., (2017) utilizaram também CCA e CCF, já o estudo Korakakis et al., (2018) utilizaram apenas em CCA, todos os três estudos obtiveram resultados em diminuição da dor anterior no joelho em pacientes com dor patelofemoral, além de terem concordado no uso de $30 \%$ de 1RM no grupo TR-OV.

Inversen et al. (2014) investigaram os efeitos da oclusão vascular em CCA com contração isométrica no pós-operatório de reconstrução de LCA, mas não houve melhora significativa nos dois grupos, obtendo o resultado que o exercício de contração isométrica sozinho não foi capaz de gerar ganhos significativos em um pós operatório de LCA. O exercício isométrico é composto basicamente por manter uma contração muscular com ângulo fixo, sendo necessário determinar o ângulo da articulação para manutenção da contração muscular, o tempo de contração e o tempo de repouso. A hipótese que por se tratar de pós-operatório de LCA os 
pacientes apresentaram um déficit acentuado na força e redução da massa muscular, devido ao processo cicatricial pós cirúrgico (PALMIERI-SMITH et al., 2008), limitando, assim, a tensão muscular gerada durante o protocolo de exercício e consequentemente diminuição da resposta neuromuscular.

Em relação a resposta hipertrófica, 88,88\% dos estudos desta revisão relatam resultados positivos no aumento do diâmetro do músculo avaliado. Ohta et al., (2003) utilizaram oclusão vascular com uma variedade de exercícios isométricos, isotônicos, e com CCA e CCF e obteve melhora no diâmetro da fibra tipo 1 e fibras tipo 2. Tennent et al. (2017) utilizaram como exercícios CCA e CCF com 30\% de 1RM e os dois grupos obtiveram melhoras funcionais e diminuição da dor, mas apenas o grupo TR-OV obteve aumento da circunferência da coxa. Os estudos de Bryk et al., (2016), Ferraz et al., (2018), Segal et al., (2015), Giles et al., (2017), Yow et al., (2017), e Korakakis et al., (2018) também relataram em seus resultados aumento da circunferência da coxa no grupo TR-OV. Essa mudança provavelmente ocorreu devido ao ambiente relativamente anaeróbico criado por um torniquete durante a execução dos exercícios. Esse ambiente, consequentemente, pode criar o estado anaeróbico necessário, com produção de metabólitos como lactato e íons $\mathrm{H}^{+}$, para permitir que os músculos produzam adaptações hipertróficas.

O aumento da força muscular foi encontrado em 77,77\% dos estudos incluídos. O estudo de Yow et al., (2017), utilizaram exercício de CCA e CCF com 30\% 1RM com oclusão vascular associado aos exercícios resistidos em dois pacientes em pós-operatório de ruptura de tendão de Aquiles. Foi observado uma melhora acelerada dos dois pacientes na força e potência muscular dos flexores plantares. Além disso, observa-se um importante benefício conquistado, um paciente voltou a pratica de corridas e o outro voltou a deambular sem auxílio de dispositivos auxiliares. Semelhante aos estudos de Ferraz et al., (2018), Segal et al., (2015), Giles et al., (2017), Tennent et al., (2017), Ohta et al (2003), e Korakakis et al., (2018) relataram aumento significativo da força muscular no grupo TR-OV. Esse aumento da força está relacionado com a resposta hipertrófica, devido ao aumento do diâmetro e aumento das fibras musculares, além do mecanismo de hipóxia que estimula maior ativação das fibras musculares tipo II, que são fibras de contração rápidas.

Por consequências as respostas positivas citadas acima, foi identificado nos achados que essas mudanças repercutiram na melhora da função dos pacientes e consequentemente na melhora da qualidade de vida. Podemos ressaltar que nos estudos avaliados, o protocolo de $30 \%$ 
de 1RM com oclusão vascular foi o mais utilizado (88,88\%). Os exercícios tanto de CCA quanto de CCF são importantes para reabilitação de lesões osteomusculares, porém deve-se ser aplicado de modo que a escolha do exercício seja de acordo com a adaptação e aceitação do paciente traçado pelo objetivo individualizado.

Em relação a intensidade do exercício foi descrita por um intervalo de repetição 15, 20 , 25, 30 ou por uma duração prolongada, não houve uma concordância nos números de repetição e séries. Os exercícios mais consistentes relatados são extensões de joelho, leg press, flexões de quadril e joelho, flexão inversa (extensão de quadril); no entanto, não existem evidências sobre o regime de exercícios preferido. Os autores ressaltam que o protocolo escolhido para serem adequados devem gerar fadiga muscular volêmica (HEITKAMP et al., 2015). A frequência de exercício relatada com TR-OV também varia muito, com relatos de 1 sessão por semana a 6 sessões por semana. A frequência pode ser menos importante que os parâmetros de exercício implementados para provocar um estado de metabolismo anabólico (NICHOLAS N. DEPHILLIPO. A. et.al., 2018).

A pressão de oclusão relatada teve variáveis diferentes, mesmo assim obteve resposta positivas. O estudo e Segal et al., (2015) realizaram pressão de 40 a $100 \mathrm{mmHg}$; Bryk et al., (2016) restringiram o fluxo sanguíneo com 200 mmHg, já os estudos de Giles et al., (2017), Korakakis et al., (2018), Ferraz et al., (2018), Tennent et al., (2017) e Yow et al., (2017) insuflaram o manguito e após eliminar o pulso detectável obteve o valor de oclusão total do fluxo, e após manteve os valores entre $60 \%$ a $80 \%$ de pressão de oclusão parcial de fluxo. Os estudos de Inversen et al., (2014), Ohta et al (2003) utilizaram pressão de $180 \mathrm{mmHg}$. Portanto a medida de pressão de oclusão mais recomendada entre os estudos foi a mínima de $40 \mathrm{mmHg}$ e a máxima de 200 mmHg. Nicholas n. Dephillipo, a. (2018) recomendaram como medida de segurança a obtenção de uma medida de oclusão arterial total em repouso e o uso de $80 \%$ dessa medida para a pressão prescrita de TR-OV específica do paciente. Outra variável diferente entre os artigos é o tamanho do manguito, atualmente a literatura relata que quanto maior a largura do manguito maior será a restrição do fluxo sanguíneo arterial a uma pressão mais baixa do que manguitos estreitos da TR-OV, que necessitam de pressões restritivas de alta magnitude (160$240 \mathrm{mmHg}$ ) para restrição de fluxo sanguíneo (NICHOLAS N. DEPHILLIPO, A. et al., 2018). Em concordância, $100 \%$ dos estudos utilizaram o manguito na região proximal da coxa.

Esta análise oferece resultado quantificado dos benefícios na melhora da dor, hipertrofia, força muscular, e da funcionalidade dos pacientes. Atualmente, ainda existem algumas outras 
variáveis, como idade, sexo, nível de condicionamento físico, status de treinamento, tipo de lesão, história pregressa, tipos de exercícios, força de base e tamanho muscular, que carecem de uma base de evidências suficiente para serem incluídas na análise, destacando a necessidade de mais estudos nessa área. No entanto, os resultados dessa análise fornecem informações sobre os benefícios e considerações metodológicas que podem ser importantes a serem consideradas em projetos de pesquisas futuras. O presente estudo identificou que os principais resultados dos artigos avaliados mostraram diminuição da dor, aumento na hipertrofia e força muscular para exercícios resistidos com resistência de baixa carga associado a oclusão vascular. Em adição, em alguns estudos também se observa melhora funcional.

Embora a TR-OV seja bem tolerado e seguro para a maioria dos indivíduos, os pacientes submetidos podem estar sujeitos a risco ao realizar os exercícios TR-OV, devido ao risco aumentado de tromboembolismo venoso (TEV) e outras patologias venosas. Embora a patogênese do TEV seja multifatorial e específica para o indivíduo, é comumente descrita como uma combinação de estase sanguínea, lesão endotelial e alterações nos constituintes do sangue, levando à hipercoagulabilidade. A literatura coletiva parece indicar que uma prescrição adequada de TR-OV apresenta pouco risco de causar diretamente um evento de TEV (BOND ET AL. 2018). Antes de prescrever a TR-OV, o profissional de saúde deve avaliar cada paciente quanto a sinais e sintomas de TEV. Segundo o estudo de Bond et al. (2018) é plausível que combinar os efeitos de uma única força compressiva do manguito da TR-OV com a natureza das contrações musculares durante o exercício resistido possa melhorar a ejeção do sangue na musculatura da extremidade inferior após o esvaziamento do manguito. Patterson et al. (2010) relataram esse efeito e sugerem que o TR-OV facilita um aprimoramento crônico na função vascular e no fluxo sanguíneo em comparação ao treinamento de resistência de baixa intensidade sozinho. Além disso, durante o exercício de TR-OV ocorrem adaptações cardiovasculares, como aumento da frequência cardíaca e da pressão arterial para sustentar o exercício. Isso significa que o fluxo sanguíneo parcial temporário é localizado no membro que realiza a TR-OV, e que o fluxo sanguíneo normal é provavelmente retomado assim que o manguito é esvaziado (BOND et al., 2018). Bioquimicamente, a rápida aceleração do sangue também aumenta a secreção de óxido nítrico das células endoteliais, dilatando os vasos e melhorando o fluxo sanguíneo (CHEN AH et al,, 2001).

Bond et al. (2018) concluíram em seu estudo que o risco de TEV associado à TR-OV é provavelmente semelhante ao do exercício resistido tradicional de alta intensidade em adultos, 
e sugere que uma prescrição adequada de TR-OV no contexto da reabilitação não aumentaria o risco de desenvolver TEV. No entanto, faz necessário que seja feito uma triagem completa dos sinais e sintomas de TEV e conhecer os fatores de risco do mesmo em cada paciente antes de prosseguir com a TR-OV, principalmente devido ao risco de TEV assintomática, deve se excluir a aplicação dos exercícios com oclusão vascular em pacientes com histórico de TEV. Os profissionais de saúde também devem garantir que tenham o treinamento adequado e que estejam usando o equipamento correto de TR-OV e as técnicas de prescrição de pressão para garantir a segurança de seus pacientes.

Assim, o exercício resistido com baixa carga associado com oclusão vascular é uma ferramenta de reabilitação clínica progressiva no processo de retorno ao exercício de carga pesada. A tensão mecânica nos pontos distais da oclusão pode explicar os estímulos para hipertrofia e aumento da força muscular. Atualmente, não há literatura de forma clara para explicar esse mecanismo fisiológico, mas a causa da hipertrofia e as adaptações neurais causadas por essa oclusão vascular é semelhante ao mecanismo observado com o que acontece no treinamento de cargas pesadas. Entretanto, com o exercício resistido de baixa carga com oclusão vascular, esse mecanismo é ativado pela combinação de tensão e hipóxia, produzindo alterações metabólicas que podem desempenhar um papel na produção de crescimento do tecido nessas situações.

Durante condições normais, as fibras de contração lenta são recrutadas primeiro, e à medida que a intensidade aumenta, as fibras de contração rápida (tipo II) são recrutadas conforme necessário. Meyer (2006) relatou que durante as condições isquêmicas, como na oclusão vascular as fibras de tipo II são recrutadas mesmo que a intensidade seja baixa. Embora não definitivas, as teorias que explicam o mecanismo ação sobre o exercício de TR-OV, é que essa mudança provavelmente ocorre devido a diminuição de oxigênio e nutrientes no membro ocluído gerando um ambiente relativamente anaeróbico criado pelo torniquete com oclusão durante os exercícios, criando requisitos anaeróbicos necessário para o aumento de hormônios anabólicos, aumento do recrutamento de fibras musculares tipo II, e aumento do inchaço celular. As unidades motoras aeróbicas, que normalmente são recrutadas com cargas leves, deverão fadigar mais rapidamente durante a restrição do fluxo sanguíneo. A eletromiografia integrada (iEMG) mostrou que a oclusão causa a ativação de um número suficiente de fibras de tipo II nessas baixas intensidades (TAKARADA et al., 2000). 
O acumulo de radicais livres e hidrogênio na corrente sanguínea gera um acúmulo de lactato no sangue total e lactato de células musculares causados devido à restrição do fluxo sanguíneo resultam em aumento de hormônio de crescimento (GH). Isso ocorre porque o GH mostrou ser estimulado por ambiente ácido produzido pelo ácido lático. Esse aumento nos níveis de GH é maior do que o normalmente observado no exercício resistido regular, o GH estimula aos receptores do fígado a produzir (fator de crescimento semelhante a insulina (IGF1) que tem papel fundamental na síntese proteica nas fibras musculares, além da alteração da oxidação lipídica (TATSUMI et al 2002). O acúmulo metabólico parece ser o principal mecanismo do exercício TR-OV. Outro fator é a contribuição da proteína de choque térmico 72 (HSP 72), do oxido nítrico sintase-1 (NOS-1) e a inibição da miostatina (LOENNEKE et al., 2009). Tatsumi et al. (2002) identificaram que a HSP 72 aumentou seus níveis durante o TR$\mathrm{OV}$, isso acontece por estressores do calor, isquemia, hipóxia e radicais livres. O HSP 72 evita a degradação e agregação de proteína, de modo que pode desempenhar um papel na hipertrofia muscular induzida por oclusão. O aumento da NOS-1 estimula ativação das células satélites, demostrando estimular o crescimento muscular.

Gundermann et al., (2014), demonstraram que a ativação das células satélites estimula a via mTORC1, tradução do mRNA e crescimento de células musculares, sendo possível a explicação da síntese de proteína muscular. Embora o mecanismo exato para a hipertrofia precoce visto após o TR-OV ainda estar sob investigação, os relatos presentes nessa revisão são importantes para explicar os fatores fisiológicos e o ponto inicial para estudos futuros.

Portanto, o exercício associado com TR-OV proporciona ganhos sem ter estresse na articulação, os pacientes toleram mais os exercícios e segundo os estudos produzindo melhoras durante o movimento (HEITKAMP et al., 2015). Portanto, essa via de hipertrofia e aumento da força possibilita a implementação precoce como reabilitação de pacientes pós-operatórios de MMII e lesões com altos índices de dor e atrofia muscular de MMII. 
Bryk et al., (2016), Ferraz et al., (2018), Segal et al., (2015), Giles et al., (2017), e Korakakis et al., (2018) investigaram exercícios com oclusão vascular parcial em pacientes com OA do joelho e dor anterior do joelho (dor patelofemoral), comparando os grupos, fisioterapia convencional altas cargas 70\% RM, baixas cargas 30\% a 50\% RM, e exercício resistido combinado com oclusão vascular com baixa carga (TR-OV) 30\% a 50\% de 1 RM. Ao usar baixas cargas, o grupo TR-OV induziu menos estresse articular resultando em diminuição do desconforto e da dor na região anterior do joelho nos cinco estudos acima, e tanto os grupos controle (alta carga) quanto os grupos TR-OV obtiveram melhoras na força e resposta hipertrófica.

Inversen et al. (2014) compararam o efeito de exercícios isométricos de baixas cargas com e sem oclusão vascular no tratamento pré e pós-operatório de reconstrução de LCA. Importante ressaltar que o grupo experimental realizou os exercícios nos intervalos da oclusão vascular. Os autores observaram que não ocorreu melhora significativa em nenhum dos grupos. Os demais estudos que utilizaram exercício isotônicos com oclusão vascular, relataram hipertrofia, aumento da força e melhora da dor como observado no estudo Ohta et al., (2003) que investigaram os efeitos do exercício isotônico com e sem oclusão vascular no pósoperatório de reconstrução de LCA e identificou aumento do diâmetro das fibras tipo 1 e tipo 2 no grupo com oclusão vascular.

Tennent et al. (2017) investigaram os efeitos do treinamento resistido com oclusão vascular no pós artroscopia de joelho comparado com exercício convencional. Foi identificado melhora da dor e força muscular nos dois grupos, mas o grupo TR-OV obteve uma melhora maior nos testes funcionais e de força quando comparado ao grupo controle. Além disso, apenas o grupo TR-OV obteve aumento significativo na circunferência da coxa.

Yow et al., (2017), pesquisaram os efeitos da oclusão vascular com exercícios resistidos no pós operatório de ruptura de tendão de Aquiles em dois pacientes. Os autores identificaram melhora da força e potência muscular dos flexores plantar de forma acelerada.

Indivíduos com lesão ligamentares, osteoarticulares e cirurgias apresentam redução do movimento, redução da massa muscular e força, o que prejudica o seu desempenho funcional (DE JONG et al., 2007). No entanto, na reabilitação nem sempre é possível a aplicação de exercício com alta carga nas fases iniciais quando a capacidade funcional do indivíduo está reduzida. Intervenções com o objetivo de promover o aumento da força e massa muscular (hipertrofia) nos momentos iniciais da reabilitação são importantes para acelerar o processo de 
recuperação nesse sentido. Os resultados dos estudos sugerem que o treinamento resistido associado com a oclusão vascular pode ser uma alternativa eficaz no tratamento precoce dos pacientes com lesões de MMII.

\section{CONCLUSÃO}

Em conclusão, os resultados da presente análise demonstram efeitos positivos na reabilitação com a técnica de TR-OV, a relevância clínica desta revisão é a demonstração de que a oclusão vascular associado com exercício resistido de baixa carga pode fornecer uma abordagem eficaz, e mais tolerável na reabilitação do que cargas pesadas, ao mesmo tempo a técnica de TR-OV minimiza a dor, melhora a função e qualidade de vida. Em relação à resposta hipertrófica, o TR-OV demonstrou induzir aumentos comparáveis na massa muscular quando comparado aos exercícios de altas cargas. Em comparação ao exercício de baixa carga sozinho o TR-OV obteve maiores respostas na força muscular, independentemente da oclusão absoluta, a prescrição de pressão, largura do manguito e pressão de oclusão, apoiando sua eficácia, apesar de diferenças nos protocolos TR-OV entre os grupos de pesquisa. A revisão discutiu alguns parâmetros necessários para o TR-OV para facilitar a implementação segura e ideal, mas pesquisas futuras devem adotar uma abordagem individualizada e progressiva para facilitar e definir um protocolo com segurança. Esse estudo deixa como base também a necessidade de estudos futuros que explique o mecanismo de adaptação que ocorre no tecido causado pela técnica de TR-OV. Entretanto, o TR-OV pode facilitar o envolvimento precoce no exercício de força e com estresse articular diminuído em uma ampla gama de populações clínicas. Portanto, nesta revisão recomenda que o TR-OV é eficaz para implementação na prática clínica de reabilitação dos pacientes com lesão musculoesquelética em MMII.

\section{REFERÊNCIAS}

AMERICAN COLLEGE OF SPORTS MEDICINE. Progression Models in Resistance Training for Healthy Adults. Medicine and Science in Sports and Exercise. v.34, n.2, p.364.380, 2002. DOI: 10.1249 / MSS.0b013e3181915670 
BOND, C. W.; HACKNEY, K. J.; BROWN, S. L.; NOONAN, B. C. Blood Flow Restriction Resistance Exercise as a Rehabilitation Modality Following Orthopaedic Surgery: A Review of Venous Thromboembolism Risk. J Orthop Sports Phys Ther. v.49, n.1, p.1.27, 2019. DOI: 10.2519 / jospt.2019.8375

CHEN, A.H.; FRANGOS, S. G.; KILARU, S.; SUMPIO, B. E. Dispositivos de compressão pneumática intermitentes - mecanismos fisiológicos de ação. Eur J Vasc Endovasc Surg. v.21, p.383-.392, 2001. DOI: 10.1053 / ejvs.2001.1348

DE JONG, S. N.; VAN CASPEL, D. R.; VAN HAEFF, M. J.; SARIS, D. B. F. Functional Assessment and Muscle Strength Before and After Reconstruction of Chronic Anterior Cruciate Ligament Lesions. Arthroscopy: The Journal of Arthroscopic \& Related Surgery., v.23, n.1, p.11.21, 2007. DOI: 10.1016 / j.arthro.2006.08.024

FERNANDES, T. L.; ANDRÉ PEDRINELLI; ARNALDO JOSÉ HERNANDEZ. Lesão muscular - fisiopatologia, diagnóstico, tratamento e apresentação clínica. Rev. bras. ortop. vol.46 no.3 São Paulo 2011. DOI: https://doi.org/10.1590/S0102-36162011000300003

FERRAZ, R. B.; GUALANO, B.; RODRIGUES, R.; KURIMORI, C. O.; FULLER, R., LIMA, F. R.; ROSCHEL, H. Benefits of Resistance Training with Blood Flow Restriction in Knee Osteoarthritis. Medicine \& Science in Sports \& Exercise., v.50, n.5, p.897.905, 2018. DOI: 10.1249 / MSS.0000000000001530

GUNDERMANN, D. M.; WALKER, D. K.; REIDY, P. T. Activation of mTORC1 signaling and protein synthesis in human muscle following blood flow restriction exercise is inhibited by rapamycin. Am J Physiol Endocrinol Metab. v.306, p.1198. 1204, 2014. DOI: 10.1152 / ajpendo.00600.2013

GUILHERME F. REIS.; THIAGO RT SANTOS.; RODRIGO CP LASMAR.; OTAVIANO OLIVEIRA JÚNIOR.; RÔMULO FF LOPES.; SÉRGIO T. FONSECA. Perfil de lesões esportivas de uma seleção brasileira de futebol da primeira divisão: um estudo de coorte descritivo. Braz. J. Phys. Ther. vol.19 no.5 São Carlos set./out. 2015 Epub 06 de outubro de 2015. DOI: https://doi.org/10.1590/bjpt-rbf.2014.0120

HEITKAMP HC. Training with blood flow restriction. Mechanisms, gain in strength and safety. J Sports Med Phys Fitness. 2015;55(5):446-456.

INGERSOLL, C. D.; GRINDSTAFF, T. L.; PIETROSIMONE, B. G.; HART, J. M. Neuromuscular Consequences of Anterior Cruciate Ligament Injury. Clinics in Sports Medicine., v.27, n.3, p.383.404, 2008. DOI: 10.1016 / j.csm.2008.03.004

KONISHI, Y.; FUKUBAYASHI, T. Relationship between muscle volume and muscle torque of the hamstrings after anterior cruciate ligament reconstruction. Journal of Science and Medicine in Sport., v.13, n.1, p.101.105, 2008. DOI: 10.1016 / j.jsams.2008.08.001

LIXANDRÃO, M. E.; UGRINOWITSCH, C.; BERTON, R. Magnitude of Muscle Strength and Mass Adaptations Between High-Load Resistance Training Versus Low-Load Resistance 
Training Associated with Blood-Flow Restriction: A Systematic Review and Meta-Analysis. Sports Med 48, p.361.378, 2018. DOI: 10.1007 / s40279-017-0795-y

LOENNEKE, J. P.; PUJOL, T. J. The Use of Occlusion Training to Produce Muscle Hypertrophy. Strength and Conditioning Journal. v.31, n.3, p.77-84, 2009. DOI: 10.1519/SSC.0b013e3181a5a352

MEYER, R.; Does blood flow restriction enhance hypertrophic signaling in skeletal muscle? J Appl Physiol. v.100, p.1443.1444, 2006. DOI: 10.1152 / japplphysiol.01636.2005

NICHOLAS N. DEPHILLIPO, A.; MITCHELL, I.; KENNEDY, B. S.; ZACHARY S. AMAN, B. A.; ANDREW, S.; BERNHARDSON, M. D.; LUKE O'BRIEN, P. T.; PHTY, S. C. S.; ROBERT, F. Blood Flow Restriction Therapy After Knee Surgery: Indications, Safety Considerations, and Postoperative Protocol. North American Arthroscopy Association, 2018. DOI: 10.1016 / j.eats.2018.06.010

PALMIERI-SMITH, R. M.; THOMAS, A. C.; WOJTYS, E. M. Maximizing Quadriceps Strength After ACL Reconstruction. Clinics in Sports Medicine., v.27, n.3, p.405.424, 2008. DOI: 10.1016 / j.csm.2008.02.001

PATTERSON, S. D.; FERGUSON, R. A. Increase in calf post-occlusive blood flow and strength following short-term resistance exercise training with blood flow restriction in young women. Eur J Appl Physiol. v.108, p.1025.1033, 2010. DOI: 10.1007/s00421-009-1309-x

PATTERSON, S. D.; HUGHES, L., HEAD, P.; WARMINGTON, S.; BRANDNER,

C. Blood flow restriction training: a novel approach to augment clinical rehabilitation: how to do it. British Journal of Sports Medicine., v.51, n.23, p.1648.1649, 2017. DOI: 10.1136 / bjsports-2017-097738. DOI: 10.1136 / bjsports-2017-097738

SATO, Y. The history and future of Kaatsu Training. Int J Kaatsu Training Res., v.1, n.1, p.5, 2005. DOI: https://doi.org/10.3806/ijktr.1.1

TAKARADA, Y., TAKAZAWA, H., SATO, Y., TAKEBAYASHI, S., TANAKA, Y., ISHII, $\mathrm{N}$. Effects of resistance exercise combined with moderate vascular occlusion on muscular function in humans. Journal of Applied Physiology., v.88, n.6, p.2097.2106, 2000. DOI: 10.1152 / jappl.2000.88.6.2097

TAKARADA, Y.; NAKAMURA, Y.; ARUGA, S.; ONDA, T.; MIYAZAKI, S. E.; ISHII, N. Rapid increase in plasma growth hormone after low-intensity resistance exercise with vascular occlusion J Appl Physiol. v.88, p.61.65, 2000. DOI: 10.1152/jappl.2000.88.1.61

TATSUMI, R.; HATTORI, A.; IKEUCHI, Y.; ANDERSON, J.; ALLEN, R. Release of hepatocyte growth factor from mechanically stretched skeletal muscle satellite cells and role of pH and nitric oxide. Mol Biol Cell. v.13, p. 2909.2918, 2002. DOI: 10.1091 / mbc.e02-010062 
THOMAS, A. C.; VILLWOCK, M.; WOJTYS, E. M., PALMIERI-SMITH, R. M. Lower Extremity Muscle Strength After Anterior Cruciate Ligament Injury and Reconstruction. Journal of Athletic Training., v.48, n.5, p.610.620, 2013. DOI: 10.4085 / 1062-605048.3.23

VAN MELICK N, VAN CINGEL RE, BROOIJMANS F, NEETER C, VAN TIENEN T, HULLEGIE W, NIJHUIS-VAN DER SANDEN MW. Evidence-based clinical practice update: practice guidelines for anterior cruciate ligament rehabilitation based on a systematic review and multidisciplinary consensus. Br J Sports Med. 2016 Dec;50(24):1506-1515. DOI: 10.1136/bjsports-2015-095898. Epub 2016 Aug 18. PMID: 27539507.

VICTOR, R.; SEALS, D. Reflex stimulation of sympathetic outflow during rhythmic exercise in humans. Am J Physiol. v.257, p.2017. 2024, 1989. DOI:

https://doi.org/10.1152/ajpheart.1989.257.6.H2017

WILLIAM, J.; KRAEMER.; KENT ADAMS.; CAFARELLI, E. Progression Models in Resistance Training for Healthy Adults. Medicine and Science in Sports and Exercise, v.34, n.2, p.364.380, 2002. DOI: 10.1097 / 00005768-200202000-00027

\section{Declaração de Interesse}

Os autores declaram não haver nenhum

conflito de interesse

\section{Financiamento Próprio}

\section{Agradecimentos}

Os autores agradecem ao Centro

Universitário Estácio de Belo Horizonte

e a Faculdade Pitágoras-Betim.

\begin{abstract}
Colaboração entre autores
Severiano: concebeu o estudo, contribuiu para o seu design, coletou os dados supervisionados, analisou e interpretou os dados, bem como escreveu o manuscrito. Inácio: concebeu o estudo, contribuiu para o seu design, coletou os dados supervisionados, analisou e interpretou os dados. Anjos: contribuiu com o conteúdo intelectual do manuscrito, interpretação dos dados e revisão da escrita do manuscrito. Rabelo: contribuiu com o conteúdo intelectual do manuscrito, interpretação dos dados e revisão da escrita do manuscrito. Ângelo: concebeu o estudo, contribuiu com o conteúdo intelectual do manuscrito, interpretação de dados e revisão da escrita do manuscrito.
\end{abstract}

